\title{
Analysis of Vibrational Resonance in Bi-harmonically Driven Plasma
}

\author{
T. O. Roy-Layinde, ${ }^{1,2}$ J. A. Laoye, ${ }^{1}$ O. O. Popoola, ${ }^{2}$ and U. E. Vincent ${ }^{3,4, a)}$ \\ 1) Department of Physics, Olabisi Onabanjo University, Ago-Iwoye, Nigeria \\ 2) Department of Physics, University of Ibadan, Ibadan, Nigeria \\ 3) Department of Physical Sciences, Redeemers' University, Ede, Nigeria \\ ${ }^{4)}$ Department of Physics, Lancaster University, Lancaster LA1 $4 Y B$, United Kingdom.
}

(Dated: 3 August 2016)

The phenomenon of vibrational resonance (VR) is examined and analyzed in a bi-harmonically driven two-fluid plasma model with nonlinear dissipation. An equation for the slow oscillations of the system is analytically derived in terms of the parameters of the fast signal using the method of direct separation of motion. The presence of a high frequency externally applied electric field is found to significantly modify the system's dynamics, and consequently, induce VR. The origin of the VR in the plasma model has been identified, not only from the effective plasma potential but also from the contributions of the effective nonlinear dissipation. Beside several dynamical changes, including multiple symmetry-breaking $(s b)$ bifurcations, attractor escapes, and reversed period-doubling bifurcations, numerical simulations also revealed the occurrence of single and double resonances induced by symmetry breaking bifurcations.

PACS numbers: Valid PACS appear here

Keywords: Vibrations, Resonances, Plasma, Oscillations

Vibrational resonance (VR) was first reported in 2000 by Landa and McClintock [J Phys A: Math \& Gen 33, L433 (2000)] in a non-linear bi-stable system driven by two external periodic forces with sharp variation in their frequencies. Since then, VR has been investigated in various model systems. In this paper, VR is examined and analyzed in a two-fluid plasma model driven by two periodic forces. The model presents an interesting scenario in which the effective nonlinear dissipation contributes significantly to the occurrence of VR, a situation that is not found in most familiar models, in particular systems with linear dissipation, such as the Duffing oscillator. Besides the analytical derivation of the equation for the slow oscillations of the system in terms of the parameters of the fast signal using the method of direct separation of motion, numerical evidence for VR is provided and its underlying dynamical mechanism examined. Furthermore, some potential industrial applications of VR in plasma system are discussed.

\section{INTRODUCTION}

Resonance, a physical concept originally developed in the context of forced oscillators is a fascinating and fundamental deterministic or stochastic phenomena exhibited by nonlinear dynamical systems and characterized by the enhancement of the maximum system's response ${ }^{1}$.

a) corresponding author: u.vincent@lancaster.ac.uk
The analysis of resonance is of broad interdisciplinary interest in terms of practical applications in physics, biology and engineering; and its occurrence can be induced by different types of external force leading to diverse form, such as stochastic resonance, vibrational resonance, coherence resonance, ghost resonance, parametric resonance, autoresonance and chaotic resonance ${ }^{1}$.

In particular, vibrational resonance ${ }^{2}$ is a phenomenon observed in non-linear systems that are driven by two external periodic forces with sharp variation in their frequencies. In such system, one of the input signals is a fast oscillation with its frequency much greater than that of the slow vibration. In VR, the noise in the stochastic resonance ${ }^{3-7}$ case or chaotic system for the chaotic resonance $^{8,9}$ is replaced by a high-frequency input signal leading to an enhancement in the system's response to variations in the parameters of the fast signal. Such amplification, termed Vibrational Resonance (VR) takes place when the response amplitude becomes minimum at the bifurcation of the effective potential.

Landa and McClintock ${ }^{2}$ first reported the occurrence of VR in the weakly damped and overdamped bistable system numerically. Thereafter, an analytical investigation to confirm VR was carried out by Gitterman ${ }^{10}$. A comprehensive analytical procedure on VR of bistable Duffing oscillator which established its effective stiffness was carried out by Blekhman and Landa ${ }^{11}$ and generalized in a book by Blekhman ${ }^{12}$. Since then, vibrational resonance has attracted a lot of attentions in bistable systems ${ }^{2,11,13}$, multistable systems ${ }^{14-16}$, excitable systems ${ }^{17}$, ratchets ${ }^{18}$, quintic oscillators ${ }^{19}$, overdamped systems ${ }^{2,15,18}$, coupled oscillators ${ }^{15,20}$, delayed systems $15,20-22$, assymetric Duffing oscillators ${ }^{23}$, fractional order damped oscillators ${ }^{24}$, feedback networks ${ }^{25}$, neuron models $17,26-28$, synthetic gene network ${ }^{29}$ and biological nonlinear maps ${ }^{30}$. Moreover, experimental evidences of vibrational resonance has also been 
reported, notably in bistable and multistable verticalcavity surface-emitting laser (VCSEL) ${ }^{13,16,31}$.

In general, nonlinear systems that are driven by low frequency signal modulated by a high frequency input signal are ubiquitous in nature and cut across different fields like neuroscience, laser physics, ionospheric physics, acoustics and atomic physics . In plasma physics, for instance, it is well understood that when neutral atoms are heated up to temperatures near or exceeding atomic ionization energies, they decompose into negatively charged electrons and positively charged ions, that are strongly influenced by each others' electromagnetic fields. Moreover, because the charges are freely moving, plasmas possess well-defined space potential and the charge assemblage are capable of undergoing collective motions of great vigor and extreme complexity. The dynamics could be modeled by a driven anharmonic oscillator; and therefore capable of exhibiting enhanced resonance oscillations in the presence of high-frequency $(H F)$ signals. Indeed, such an assemblage known as plasma has been well investigated due to its numerous applications in technologies ${ }^{32}$.

Different techniques are needed to model the plasma sources, the chemical and surface interactions, and the microscopic processes in the etching of patterns. The plasma is usually modelled with hybrid codes which treat them as a fluid except where particle kinetics is necessary, such as in reactive collisions ${ }^{32,33}$. In this direction, several models have been proposed. Here, we will focus on the strongly nonlinear dissipative magnetized plasma model ${ }^{34,35}$ which describe plasma as consisting of electrons and ions constructed from a set of quasihydrodynamic equation and investigate the occurrence of VR when $H F$ signal is imposed on the system. Despite the recent burst of research activities ${ }^{36-38}$ and the importance of plasma in communication and human activities in general, the possibility of observing VR in dissipative plasma models driven by two periodic forces with its possible implications are yet to be explored. In this paper, we theoretically and numerically examine and analyze VR in a plasma model. We identified its origin, not only from the effective plasma potential but also from the contributions of the effective nonlinear dissipation. The rest of the paper is organized as follows: In section II, the model is presented. A detailed analytical description of the resonance behaviour is given in section III. Numerical simulation is presented in section IV; while the paper is concluded in section $\mathrm{V}$.

\section{THE MODEL}

A convenient model system for this study is the two fluid model that treats plasma as two inter-penetrating conducting fluids of positive ion $(i)$ with charge $+e$ and electron $(e)$ with charge $-e$. In general, the Eulerian equations which is analogous to the equations of hydro-

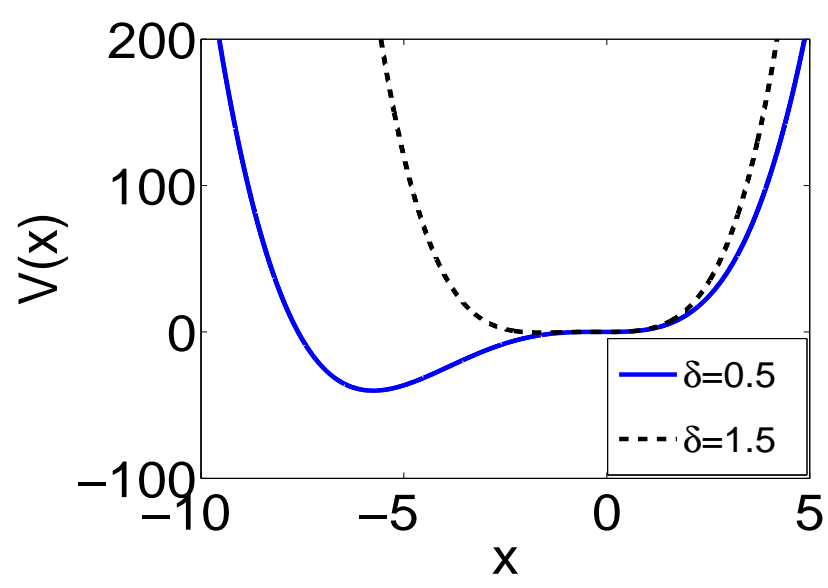

FIG. 1. The asymmetric double well potential of the dimensionless bi-harmonically driven plasma oscillations for $\delta=0.5$ and $\delta=1.5$ with parameters: $\kappa=3.05$ and $\omega_{o}=1$ fixed.

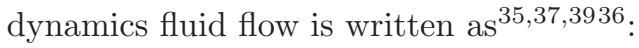

$$
\begin{aligned}
& n_{\alpha} M_{\alpha} \frac{d v_{\alpha}}{d \tau}=n_{\alpha} q_{\alpha}\left(E+v_{\alpha} \times B-\mu J\right)-\Delta P_{\alpha}, \\
& \frac{\partial n_{\alpha}}{\partial \tau}+\Delta \cdot\left(n_{\alpha} v_{\alpha}\right)=S \\
& \frac{d}{d \tau}\left(P_{\alpha} n_{\alpha}^{-\gamma}\right)=0
\end{aligned}
$$

where $S$ denotes the effect of ionization or large amplitude vibrations. The indexing for each of the species $(i$ and $e$ ) is denoted by $\alpha . n_{\alpha}$ is the density with respect to the species; $v_{\alpha}$ and $P_{\alpha}$ represent their velocity and pressure, respectively, $\gamma$ is the specific heat ratio, and $\mu_{\alpha}=$ $M_{\alpha} \nu_{\alpha} / n_{\alpha} e^{2}$ is the resistive collision with $\nu_{\alpha}$ being the collision frequency. $M_{\alpha}$ denotes the mass of each specie $\alpha$. Model (1) has attracted lots of research interests as it represents high density plasma interactions with high frequency electromagnetic waves. It finds applications in reactive ion etchers, helicon thrusters, transformer coupled plasmas, inductively coupled plasmas, electron cyclotron resonance (ERC) plasma sources and plasmas resulting from strong explosions in the atmosphere ${ }^{32}$. Thus, system (1) is very appropriate for the analysis of vibrational resonance because high frequency driving force modulates slow signals transporting the ions. It is noteworthy that photo-detachment process can physically represent a slow external driving mechanism which controls the charge on the particle while externally applied electric field with a large periodic potential may be ideal for the high frequency drive which can only be induced by transformer action in a toroidal system ${ }^{35}$.

Enjieu et al. ${ }^{35,37,39}$ had shown explicitly that the plasma oscillation may be modeled by a periodically driven nonlinear anharmonic oscillator as follows: 


$$
\begin{aligned}
\frac{d^{2} n_{1}}{d \tau^{2}} & +\left(v_{i}+2 \lambda+3 \mu n_{1}^{2}\right) \frac{d n_{1}}{d \tau} \\
& +\omega^{2} n_{1}+v_{i}\left(\lambda n_{1}^{2}+\mu n_{1}^{3}\right)=F \cos \Omega \tau
\end{aligned}
$$

Re-scaling the variables in Eq. (2) as $t=\omega_{0} \tau, n_{1}=$ $\left(\frac{v_{i}+2 \lambda}{3 \varepsilon}\right) x=\Xi x, \epsilon=\frac{v_{i}+2 \lambda}{\omega_{0}}, \kappa=\frac{v_{i} \lambda \Xi}{\omega^{2}}, \delta=\frac{v_{i} \mu \Xi^{2}}{\omega_{0}^{2}}, f=$ $\frac{F}{\Xi \omega_{0}^{2}}$ and $\Omega=\frac{\omega}{\omega_{0}}$, the dimensionless form of Eq. (2) now becomes

$$
\ddot{x}+\varepsilon\left(1+x^{2}\right) \dot{x}+\frac{d V(x)}{d x}=f \cos \Omega t ;
$$

with $V(x)$ shown in Fig. 1 being the asymmetric plasma potential given $\operatorname{as}^{36,38}$

$$
V(x)=\frac{\omega_{0}^{2}}{2} x^{2}+\frac{\kappa}{3} x^{3}+\frac{\delta}{4} x^{4} .
$$

The dots represent the differentiation with respect to time $(t), \mu, \omega_{0}, \Omega$ and $F$ are the damping coefficients, fundamental frequency, forcing frequency and amplitude of the external force respectively, $\kappa$ and $\delta$ are quadratic and cubic nonlinearity parameters, respectively.

\section{ANALYTICAL DESCRIPTION OF RESONANCES}

Here, we use the method of direct separation of motions described by Blekhman ${ }^{12}$ as the most effective formulation of vibrational mechanics to obtain the equation of the slow motion which can be modulated by parameters of the fast driving signal analytically. The biharmonically driven anharmonic plasma oscillations form of the Eqn.(3) can be expressed as

$$
\ddot{x}+\varepsilon\left(1+x^{2}\right) \dot{x}+\frac{d V(x)}{d x}=f \cos \omega t+g \cos \Omega \tau
$$

where $f \cos \omega t$ is the low frequency input signal while $g \cos \Omega \tau$ represents the high frequency input signal. For a long time $\Omega \gg \omega$ and $\tau=\varepsilon t$, we seek solution of Eqn.(5) such that

$$
x(t)=\chi(t)+\psi(t, \tau) .
$$

If $\psi$ is a periodic function with period $\frac{2 \pi}{\Omega}$ and its mean value w.r.t fast time $\tau$ is given by

$$
\langle\psi\rangle=\bar{\psi}=\frac{1}{2 \pi} \int_{0}^{2 \pi} \psi d \tau=0 ;
$$

then, the aim is to obtain two systems of integraldifferential equations from Eqn.(5) such that if a pair $(\chi, \psi)$ is a solution to the two integro-differential equations, then $x=\chi+\psi$ factoring in Eqn.(7) completely solves Eqn.(5). Thus, substituting Eqn.(7) into Eqn.(5), we have

$$
\begin{gathered}
\ddot{\chi}+\varepsilon\left(1+\chi^{2}+2 \chi \psi+\psi^{2}\right) \dot{\chi}+\left(2 \varepsilon \psi \dot{\psi}+\omega_{0}^{2}+\kappa \psi+3 \delta \psi^{2}\right) \chi \\
+(\epsilon \dot{\psi}+\kappa+3 \delta \psi) \chi^{2}+\delta \chi^{3}+\ddot{\psi}+\varepsilon\left(1+\psi^{2}\right) \dot{\psi}+\omega_{0}^{2} \psi+\kappa \psi^{2} \\
+\delta \psi^{3}=f \cos \omega t+g \cos \Omega \tau .
\end{gathered}
$$

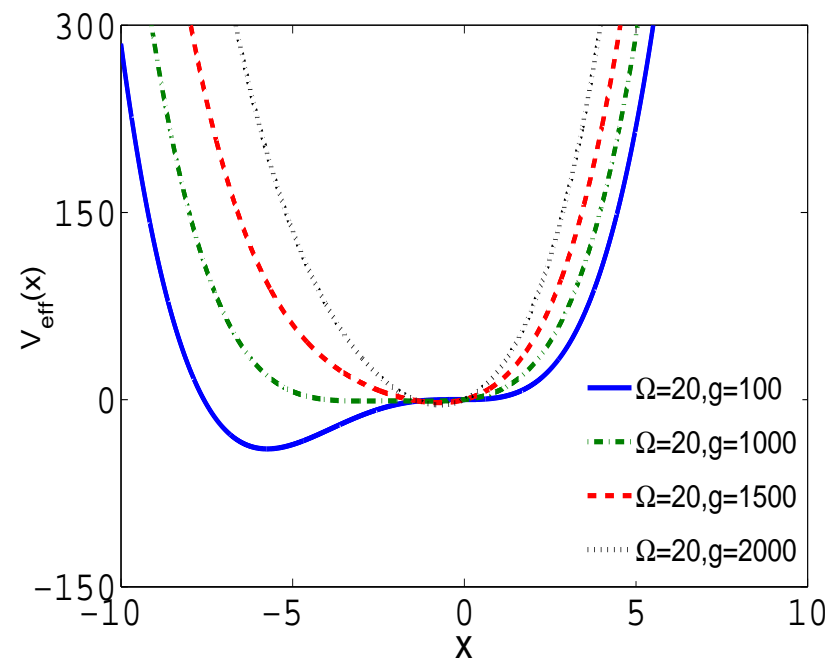

FIG. 2. The effective potential corresponding to slow motion of the system for four values of fast amplitude; $g=$ 100,1000, 1500 and 2000 for parameter values: $\delta=1.5$, $\kappa=3.05$ and $\omega_{o}=1, \Omega=20$ and $\omega=1$

Using Eqn.(7) and averaging Eqn.(8) w.r.t. fast time $\tau$, we have

$$
\begin{gathered}
\ddot{\chi}+\varepsilon\left(1+\chi^{2}+\chi \bar{\psi}+\bar{\psi}^{2}\right) \dot{\chi}+\left(2 \varepsilon \bar{\psi} \overline{\dot{\psi}}+\omega_{0}^{2}+2 \kappa \bar{\psi}+3 \delta \bar{\psi}^{2}\right) \chi \\
+(\epsilon \overline{\dot{\psi}}+\kappa+3 \delta \bar{\psi}) \chi^{2}+\delta \chi^{3}+\overline{\ddot{\psi}}+\varepsilon\left(1+\bar{\psi}^{2}\right) \overline{\dot{\psi}}+\omega_{0}^{2} \bar{\psi}+\kappa \bar{\psi}^{2} \\
+\delta \bar{\psi}^{3}=f \cos \omega t+g \cos \Omega \tau,
\end{gathered}
$$

which yield

$$
\begin{gathered}
\ddot{\chi}+\varepsilon\left(1+\chi^{2}+\bar{\psi}^{2}\right) \dot{\chi}+\left(\omega_{0}^{2}+3 \delta \bar{\psi}^{2}\right) \chi+\kappa \chi^{2}+\delta \chi^{3}+\kappa \bar{\psi}^{2} \\
+\delta \bar{\psi}^{3}=f \cos \omega t
\end{gathered}
$$

Subtracting Eqn.(10) from Eqn. (8), we have

$$
\begin{gathered}
\ddot{\psi}+\varepsilon\left(1+(\psi+\chi)^{2}\right) \dot{\psi}+\omega_{0}^{2} \psi+\kappa\left(\psi^{2}-\bar{\psi}^{2}\right)+\delta\left(\psi^{3}-\bar{\psi}^{3}\right) \\
+\varepsilon \dot{\chi}\left(\psi^{2}-\bar{\psi}^{2}\right)+3 \varepsilon \dot{\chi} \chi(\psi-\bar{\psi})+2 \kappa \chi(\psi-\bar{\chi}) \\
+3 \delta \chi\left(\psi^{2}-\bar{\psi}^{2}\right)=g \cos \Omega \tau .
\end{gathered}
$$

Eqns.(10) and (11) are the required integro-differential equations of motion representing the slow motion $\chi$ and the fast motion $\psi$, respectively. Our interest lie in Eqn.(10) which is the equation of motion of the slow dynamics of system that can be modulated appropriately by changing the parameters of the fast signal to verify the existence of VR. In this regards, we assume that the $\psi$ component is much more faster than the slow component $\chi$, such that the components $\chi$ and $\dot{\chi}$ are considered frozen i.e. constant in Eqn.(11). Thus, Eqn.(11) can be approximated using inertial approximation $\ddot{\psi} \gg \dot{\psi} \gg \psi$... to

$$
\ddot{\psi}=g \cos \Omega t
$$

which has a solution

$$
\psi=\frac{-g}{\Omega^{2}} \cos \Omega t
$$


so that $\bar{\psi}^{2}=\frac{g^{2}}{2 \Omega^{4}}, \bar{\psi}^{3}=0$. Using Eqn.(13) in Eqn.(10), we can write

$\ddot{\chi}+\varepsilon\left(1+\chi^{2}+\frac{g^{2}}{2 \Omega^{4}}\right) \dot{\chi}+\left(\omega_{0}^{2}+\frac{3 \delta g^{2}}{2 \Omega^{4}}\right) \chi+\kappa \chi^{2}+\delta \chi^{3}+\kappa \frac{g^{2}}{2 \Omega^{4}}=f \cos \varsigma$

Re-scaling Eqn.(14), we can write

$$
\ddot{\chi}+\varepsilon\left(C_{1}+\chi^{2}\right) \dot{\chi}+C_{2} \chi+\kappa \chi^{2}+\delta \chi^{3}+C_{3}=f \cos \omega t
$$

where

$$
C_{1}=1+\frac{g^{2}}{2 \Omega^{4}}, C_{2}=\omega_{0}^{2}+\frac{3 \delta g^{2}}{2 \Omega^{4}}, C_{3}=\kappa \frac{g^{2}}{2 \Omega^{4}}
$$

The effective potential corresponding to the slow motion of the system described by Eqn.(10) is

$$
V_{\text {eff }}=\frac{C_{2}}{2} \chi^{2}+\frac{\kappa}{3} \chi^{3}+\frac{\delta}{4} \chi^{4}+C_{3} \chi .
$$

Fig. 2 shows the plot of the effective potential $V_{\text {eff }}$ against the component of the slow motion, $\chi$ for $g=$ $100,1000,1500$ and 2000 with parameters $\delta=1.5, \kappa=$ $3.05, \omega_{o}=1$ and $\Omega=20$. When $g=100$, the effective potential is a double well with appreciable resemblance to the system's potential shown in Fig. 1. As the value of $g$ is increased, the shape of the potential changes and at $g=$ 2000 , the $V_{\text {eff }}$ becomes a single well with no resemblance to the system's potential presented in Fig. 1. Here, we can see that the effective potential of the slow motion depend on the parameters of the fast motion $\Omega$ and $g$. Moreover, if the high frequency signal dies out, by setting the value of the fast amplitude $g$ to zero in Eqn.(14), Eqn.(15) is reduced to the model of the harmonically driven plasma presented by Enjieu Kadji et al. ${ }^{35}$

Interestingly, from Eqn.(14) there is also a contribution to the effective dissipation of the system due to the parameters of the fast signal oscillations. The effective non-linear dissipation of the system is thus $\varepsilon\left(1+\chi^{2}+\frac{g^{2}}{2 \Omega^{4}}\right)$. This change in the effective dissipation of the system is not found in systems with linear dissipation such as the asymmetric Duffing oscillator which has been well studied for VR in both the underdamped and overdamped cases $^{23}$. The effective dissipation can play a direct role just like the effective potential in the enhancement of signals by modulating the parameters of the fast signal. These parameters therefore dictate the equilibrium points of the slow motion; which can be computed from the equation

$$
C_{3}+C_{2} \chi+\kappa \chi^{2}+\delta \chi^{3}=0 .
$$

The number of equilibrium states for the effective potential has increased to four equilibrium points $X^{*}$ for the potential $V_{\text {eff }}(x)$ from the three stationary points of $V(x)$ when $\delta>0, \kappa>0$. We can describe the oscillations in terms of deviations from the equilibrium points. For such deviation of slow motion of $\chi$ from $\chi^{*}$, using a change of variable $Y=\chi-\chi^{*}$ in Eqn.(15) we obtain

$$
\begin{array}{r}
\ddot{Y}+\alpha_{1} \dot{Y}+\varepsilon\left(Y+\chi^{*}\right)^{2} \dot{Y}+\alpha_{2} Y+\alpha_{3} Y^{2} \\
+\delta Y^{3}+\alpha_{4}=f \cos \omega t
\end{array}
$$

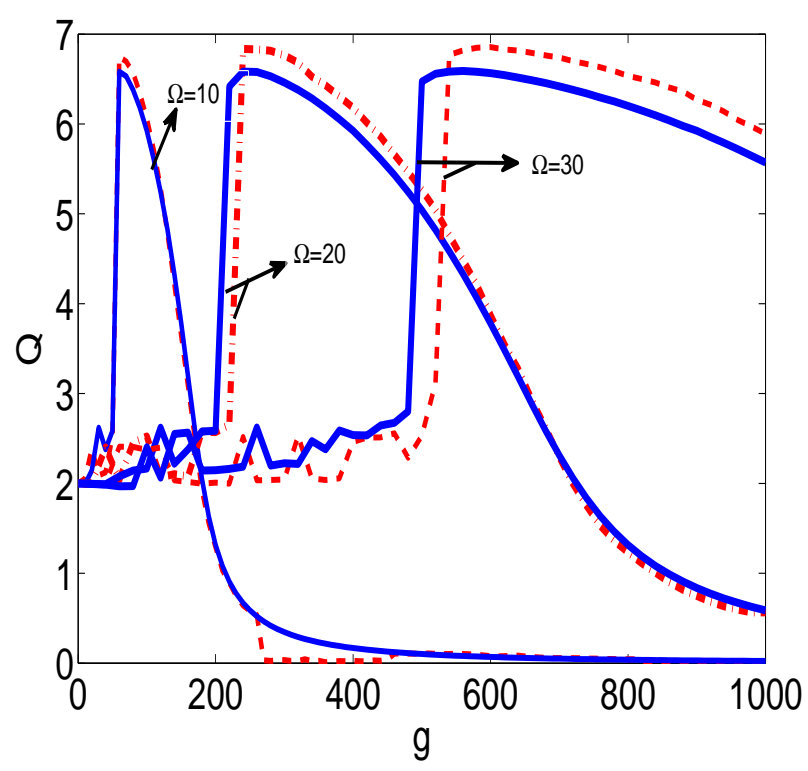

FIG. 3. Dependence of $Q$ on $g$ for $\Omega=10, \Omega=20$ and $\Omega=$ 30. Analytical (solid lines) calculated from Eqn. (18) and numerical (broken lines) from Eqn. (5). Parameters are: $\delta=$ $0.3, \kappa=1.0, \varepsilon=0.04, \omega_{0}=1, f=0.3, \omega=1$.

where $\alpha_{1}=\varepsilon C_{1}, \alpha_{2}=C_{2}+2 \kappa \chi^{*}+3 \delta \chi^{* 2}, \alpha_{3}=\kappa+3 \delta \chi^{*}$, $\alpha_{4}=C_{2} \chi^{*}+\kappa \chi^{* 2}+\delta \chi^{* 3}$. For $f \ll 1$, one can assume that $|Y| \ll 1$. Thus, by neglecting the nonlinear term in Eq. (18), we obtain a linear system written as

$$
\ddot{Y}+\left(\alpha_{1}+\varepsilon \chi^{* 2}\right) \dot{Y}+\alpha_{2} Y=f \cos \omega t-\alpha_{4} .
$$

Eq.(19) has a solution $Y(t)=A_{L} \cos (\omega t+\phi)$ in the limit $t \rightarrow \infty$; and the response amplitude $A_{L}$ is given as

$$
A_{L}=\frac{f}{\sqrt{\left(\omega_{r}^{2}-\omega^{2}\right)^{2}-\gamma^{2} \omega^{2}}}
$$

where $\omega_{r}=\sqrt{\alpha_{2}}$ is the resonant frequency of the linear system (19) and $\gamma=\alpha_{1}+\varepsilon \chi^{* 2}$. The amplitude of the system's response is thus $Q=\frac{A_{L}}{f}$.

In systems with linear dissipation, $\gamma$ appearing in Eqn. (20) is the linear damping coefficient. However, in our system, the parameter $\gamma=\varepsilon C_{1}=\varepsilon\left(1+\frac{g^{2}}{2 \Omega^{4}}\right)$ is dependent on both the frequency, $\Omega$ and amplitude, $g$ of the fast signal. Thus, a variation in the parameters of the fast signal ( $g$ or $\Omega$ ) produces additive and multiplicative effects on the appearance of VR - which is the hallmark of this paper. Now, setting $W=\omega_{r}^{2}-\omega^{2}$ and $S=\left(\omega_{r}^{2}-\omega^{2}\right)^{2}+\gamma^{2} \omega^{2}$, we find that $Q$ achieves its maximum value when $S$ is minimum, i.e., when $W=0$. Resonance would therefore occur if $\omega_{r}=\omega$, where $\omega=\sqrt{\alpha_{2}}=$ $C_{2}+2 \kappa X^{*}+3 \delta X^{*}$. If the oscillation takes place around the equilibrium $X^{*}=0$, then, $\omega=\sqrt{C_{2}}$.

Additionally, it is easy to deduce from $\gamma$ two extreme cases that our system could also satisfy. 


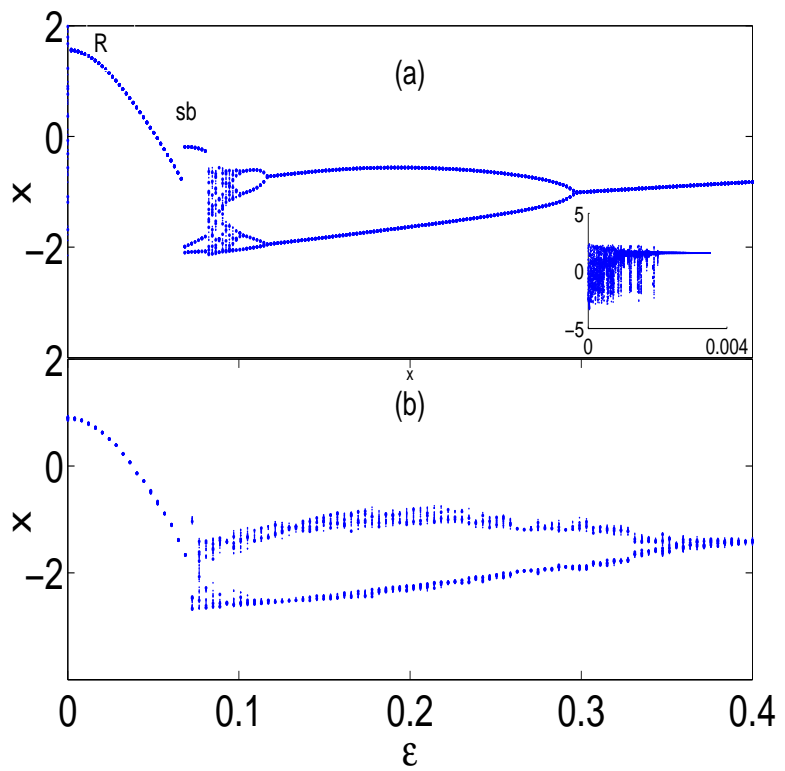

FIG. 4. Bifurcation diagram as a function of $\varepsilon$ showing (a) a resonance orbit $(\mathrm{R})$, symmetry breaking $(\mathrm{sb})$ and reversed period-doubling bifurcations for $g=0$ and (b) the deformation of bifurcation structure in (a) when $g=500$. Inset is the narrow chaotic band near $\varepsilon=0$ in (a). Parameters are: $\delta=0.3, \kappa=1, \omega_{0}=1, \omega=0.1, f=0.5, \Omega=3$.

(i) Case 1: $g$ and $\varepsilon$ fixed, with $\Omega$ varying. In this case, when $\Omega \rightarrow \infty, \gamma=\varepsilon$, the system reduces to a Duffing-type oscillator with linear dissipation; and when $\Omega \rightarrow 0, \varepsilon=0$, it becomes a conservative system.

(ii) Case 2: $\Omega$ and $\varepsilon$ fixed, with $g$ varying. In this case, when $g \rightarrow \infty, \varepsilon=0$, and it becomes a conservative system. However, when $g \rightarrow 0, \gamma=\varepsilon$. Then, we have an oscillator with linear dissipation.

\section{NUMERICAL RESULTS}

In order to numerically integrate the plasma system (5), it is convenient to express it as a set of two coupled autonomous ODEs in the form

$\frac{d x}{d t}=y$

$\frac{d y}{d t}=-\varepsilon\left(1+x^{2}\right) y-\omega_{o}^{2} x-\kappa x^{2}-\delta x^{3}+f \cos \omega t+g \cos \Omega \tau$.

These equations were integrated using the Fourth-Order Runge-Kutta (FORK) scheme with fixed step sizes $\Delta t=$ 0.001. The following parameters were fixed throughout the paper $\delta=0.3, \kappa=1, \omega_{0}=1, \omega=0.1$. The initial conditions are $x(0)=1, y(0)=1$ and the total time is $\mathrm{t}$ $=4000$ with the first 100 iterates dropped as transients.

We examined VR by investigating the linear response $Q$ of the dissipative plasma at the lower frequency input signal. $Q$ was computed from the expression

$$
Q=\frac{\sqrt{B_{S}^{2}+B_{C}^{2}}}{f}
$$

and the corresponding phase shift $\phi$ defined as

$$
\phi=-\tan ^{-1}\left(\frac{B_{S}}{B_{C}}\right)
$$

where $T=\frac{2 \pi}{\Omega}, n(=1,2,3 \ldots)$ is any positive integer.

$$
\begin{aligned}
& B_{S}=\frac{2}{n T} \int_{0}^{n T} x(t) \sin \omega t d t \\
& B_{C}=\frac{2}{n T} \int_{0}^{n T} x(t) \cos \omega t d t .
\end{aligned}
$$

In order to examine the amplitude response of the system, Eqns. (22) and (23) were computed from the numerically integrated Eqn. (24) using the earlier obtained solutions of the bi-harmonically driven plasma of Eqn. (3). To validate the analysis in Sec. III, theoretical $Q$ was obtained by numerically integrating Eqn. (18) and the results compared with $Q$ obtained directly from Eqn. (22). Fig. 3 shows the plot of $Q$ for three values of $\Omega$ (10, 20 and 30) computed from Eqn. (18) and superimposed with their corresponding numerical curves computed from Eqn. (5) for comparison. One can obviously see that the theoretical and numerical results are in close agreement, with all curves having nearly equal peaks. However, the position of the peaks are shifted further away from the origin as the value of $\Omega$ increases.

Next, we investigate the effect of the nonlinear dissipation on VR. We begin by examining the changes in the system's dynamics as the coefficient of the nonlinear dissipation $\varepsilon$ is varied for $g=0$ and $g=500$; while other parameters are fixed as follows: $\delta=0.3, \kappa=1, \omega_{0}=$ $1, \omega=0.1, f=0.5$, and $\Omega=3$. Fig. 4 shows a bifurcation diagram as a function of $\varepsilon$ which was not reported in previous studies in plasma dynamics ${ }^{35,38}$. Here, a distinct sequence of bifurcation, including reversed perioddoubling bifurcations take place (Fig. 4(a)) when $g=0$ - corresponding to a system with one external forcing. For weak values of $\varepsilon$ (typically, $\varepsilon<0.003$ ), a narrow chaotic zone exists where the system occupies with interconnected chaotic orbit (See the inset in Fig. 4(a)). As the dissipation coefficient is further increased, the chaotic orbit bifurcates and dissolves into a resonating periodic orbit $(\mathrm{R})$ which undergoes a symmetric-breaking bifurcation at $\varepsilon \approx 0.068$ in which three coexisting periodic orbits are born. Further increase in $\varepsilon$ to $\varepsilon \approx 0.08$ drives the system to another short-lived chaotic state which on further increase of $\varepsilon$ experiences a reversed period-doubling to a periodic orbit. For $g=500$ (the biharmonically driven case) shown in Fig. 4(b), the narrow chaotic band at $0 \leq \varepsilon \leq 0.003$ in Fig. 4(a) disappears; while the distinct and smooth bifurcation structure immediately after the 

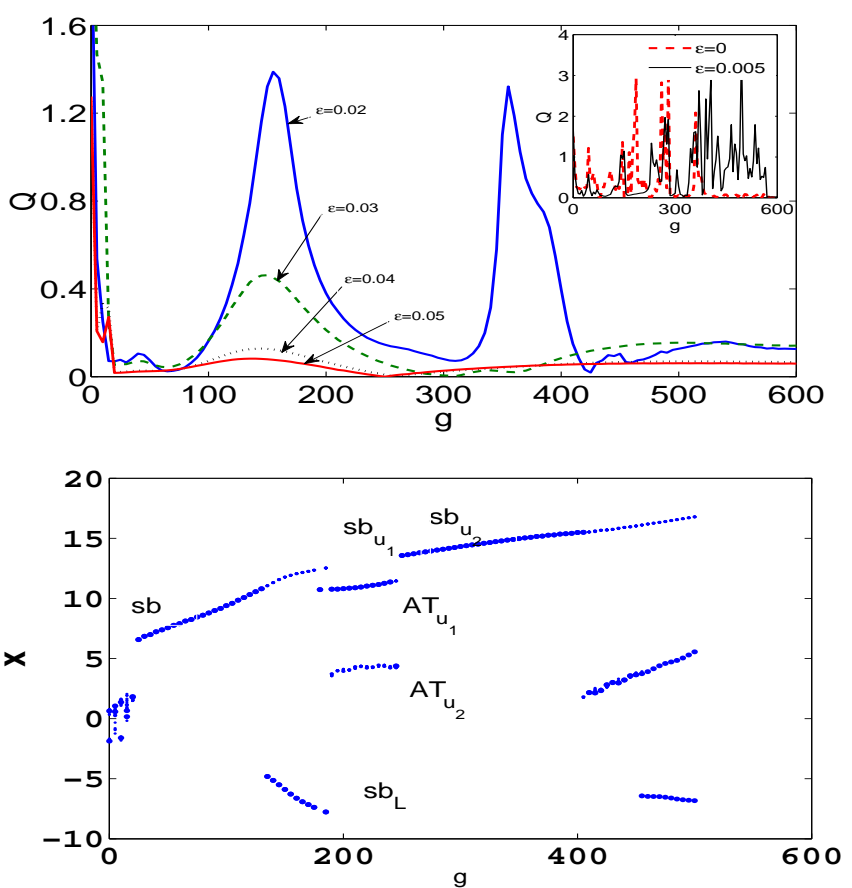

FIG. 5. Dependence of $Q$ on $g$ for different values of the nonlinear dissipation coefficient, $\varepsilon$ (Upper panel) and corresponding bifurcation diagram as a function of $g$ for $\varepsilon=0.02$ (Lower panel) illustrating the occurrence of symmetry-breaking bifurcations. Inset is for weak values of dissipations. Parameters are: $\delta=0.3, \kappa=1, \omega_{0}=1, \omega=0.1, f=0.5$, and $\Omega=3$.

occurrence of chaos at $\varepsilon \approx 0.08$ experiences some deformation with a corresponding widening of the bifurcation branches. Interestingly, whereas the resonating periodic attractor in the interval $0 \leq \varepsilon<0.06$ survives the deformation and control effects of the fast signal, $g \cos \Omega t$, its oscillation is however enhanced through the process of vibrational resonance as we shall show.

In what follows, we focus on the parameter regime, $0 \leq \varepsilon<0.06$ corresponding to the $\varepsilon$ range where the resonating attractor was observed in Fig. 4(a) to illustrate the effect of gradual variation in the coefficient of dissipation $\varepsilon$ on VR. Fig. 5(Upper panel) shows that $Q$ exhibits one or two distinct peaks, namely, single and double resonances, respectively for $0.02 \leq$ $\varepsilon \leq 0.05$. In fact, for $\varepsilon=0.02$, five resonant peaks denoted as $Q_{i}\left(g_{i}, Q_{\max }\right),(i=1, . ., 5)$ are observable: $Q_{1}(40,0.11), Q_{2}(155,1.39), Q_{3}(355,1.32), Q_{4}(450,0.11)$ and $Q_{5}(535,0.16)$. Notice that $Q_{2}(155,1.39)$ and $Q_{3}(355,1.32)$ are distinct with their amplitudes almost equal and $Q_{3}$ occurring at $g_{3}=3 g_{1}$. The two adjacent peaks to $Q_{2}(155,1.39)$ and $Q_{3}(355,1.32)$, that is, $Q_{1}(40,0.11)$ and $Q_{4}(450,0.11)$ are of equal and suppressed amplitude, relative to $Q_{2}(155,1.39)$ and $Q_{3}(355,1.32)$. We shall look closely at the dynamical mechanism associated with this amplitude suppression later. In this regime, a steady decrease in $\varepsilon$ results in rapid increase in the response amplitude $Q$. At very
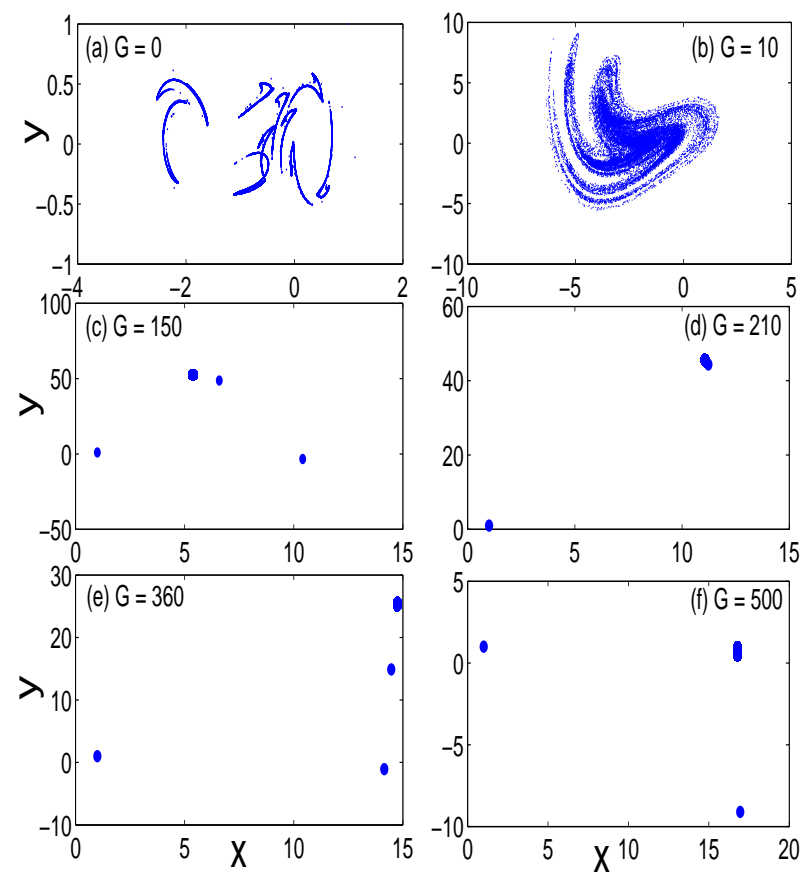

FIG. 6. Attractors in $(x, y=\dot{x})$ phase space showing the Poincaré plots of the various dynamical changes taking place during the multiple - $(s b-s b)$ - bifurcations $g=$ $0,10,150,210,360$ and 500 for figures a,b c, d, e and $\mathrm{f}$ respectively. Parameters are: $\delta=0.3, \kappa=1, \omega_{0}=1, \omega=0.1$, $f=0.5, \Omega=3$ and $\varepsilon=0.02$.

small values of dissipation coefficient corresponding to the narrow chaotic band in Fig. 4(a), $Q$ typically becomes turbulent and enhancement or depression of resonance becomes challenging with the emergence of sharp spikes that are apparently connected to chaotic dynamics. This is shown in the inset of Fig. 5(Upper panel). Furthermore, when $\varepsilon=0$ Eqn. (5) reduces to the conservative plasma model with high frequency excitation ${ }^{39}$,

$$
\ddot{x}+\omega_{0}^{2} x+\beta x^{2}+\alpha x^{3}=f \cos \omega t+g \cos t \Omega t .
$$

In this case, VR does not occur for the parameter values used here as can be seen in the inset of Fig. 5, rather $Q$ shows several spikes, indicating that the system is in a chaotic state. The emphasis here is that beside the role of the system's effective potential (16), the effective nonlinear dissipation, $\gamma$ of the system plays a critical contributory role in the occurrence of VR in this plasma model. Remarkably, the enhancement of signal by means of VR in plasma is particularly significant with a given set of carefully chosen low-dissipation.

It has been well established that resonance curves are closely connected to the underlying global bifurcation set of nonlinear oscillators ${ }^{1,40,41}$. In addition, Kozłowski et al. ${ }^{41}$ had reported that symmetric-breaking $(s b)$ bifurcations occur between resonances. Therefore, to uncover the dynamical mechanism associated with the occurrence of double-resonance and amplitude suppression, we plotted in Fig. 5(Lower panel) the bifurcation diagram for 

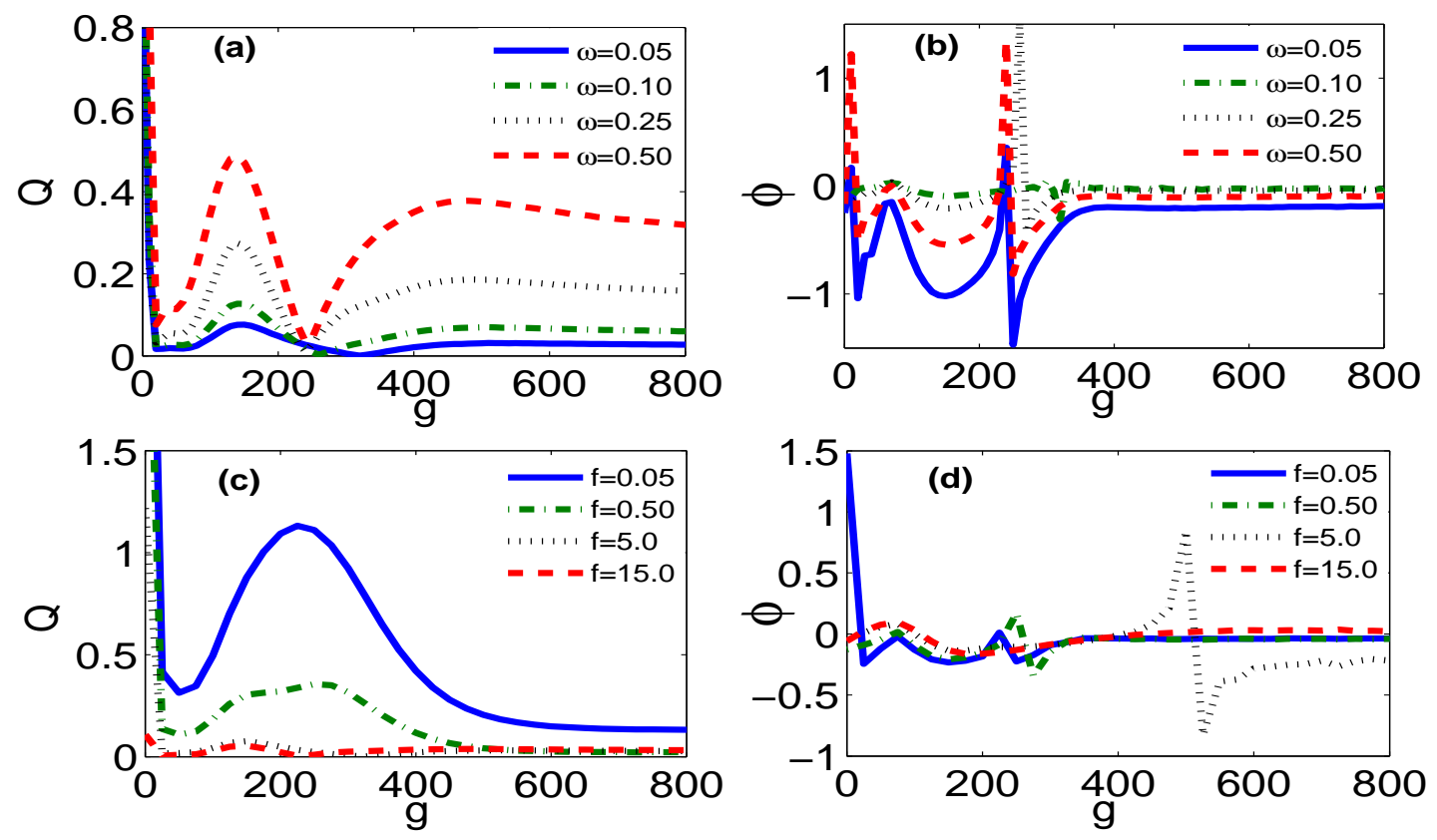

FIG. 7. System's response, Q to fast amplitude $g$ and the corresponding phase shift $\phi$ for parameters $\delta=0.3, \kappa=1.0, \omega_{0}=$ $1.0, \varepsilon=0.04$. (a) and (b) $f=0.5$ and $\omega=0.05,0.1,0.25$, and 0.5. (c) and (d) $\omega=0.1$ and $f=0.05,0.5,5.0$ and 15.0

$\varepsilon=0.02$ where pronounced double resonance appeared. Notice here that when the driving amplitude, $g$ of the fast signal is increased, multiple symmetry breaking $(s b)$ bifurcation takes place alongside the appearance of several resonances.
Hereafter, we adopt the notation $-(s b-s b)-$ which implies that two parameter values exist where symmetrybreaking bifurcations occur, the first being where a symmetrical periodic attractor splits into coexisting attractors, and the second corresponding to the point at which the coexisting attractors merge to form symmetrical periodic attractor ${ }^{41,42}$. In Fig. 5(Lower panel), four $s b$ bifurcations can be observed. The first $s b$ occur simultaneously near $g=25$, given birth to two $2 T$ periodic attractors at the lower and upper branches of the bifurcation diagram for $g<185$ as shown in Fig. 5. At $g \approx 185$, the two branches simultaneously undergo $s b$ bifurcations, denoted by $s b_{u_{1}}$ and $s b_{L}$, respectively; each splitting into two attractors. Whereas the upper branch splits into $A T_{u_{1}}$ and $A T_{u_{2}}$ that recombines at $g \approx 250$ $\left(s b_{u_{2}}\right)$, the lower branch attractor experience an escape dynamics ${ }^{43,44}$ during the $s b_{L}$ bifurcations scenario and varnishes for some range of $g$ values. Fig. 5(Lower panel) shows that attractor escape occurs at two regimes in the lower $s b$ bifurcation branch, first at $g \approx 15$, corresponding to the first suppressed resonance peak, $Q_{1}(40,0.11)$; and secondly at $g \approx 185$, corresponding to the second suppressed resonance peak, $Q_{4}(450,0.11)$. Depending on the stability of the attractors born at $g \approx 25$, the system could follow either the lower or upper symmetry bifurcation branch ${ }^{40}$. Thus, comparing the Upper panel ( $Q$ vs $g$ ) and the Lower panel ( $x$ vs $g$ ) plots in Fig. 5 , it is evident that $s b_{u_{1}}$ and $s b_{u_{2}}$ points as well as the attractors $A T_{u_{1}}$ and $A T_{u_{2}}$ are sandwiched by the two well pronounced resonance peaks, $Q_{2}(155,1.39), Q_{3}(355,1.32)$ in the Upper panel. The system's dynamics is apparently dominated by the upper symmetry-breaking bifurcation branch for increasing $g$, implying that $-(s b-s b)$ - bifurcations account for the occurrence of double-resonance peaks. At the lower symmetry bifurcation branch, attractor escape appears to be responsible for the suppression of the adjacent peaks $Q_{1}(40,0.11)$ and $Q_{4}(450,0.11)$.

To complete this picture, and starting with a transient chaotic attractor at $g=0$, Fig. 6 shows the Poincaré plots of the various dynamical changes in phase space accompanying the multiple $s b$ bifurcations for the parameters set: $\delta=0.3, \kappa=1, \omega_{0}=1, \omega=0.1, f=0.5$, $\Omega=3$ and $\varepsilon=0.02$; and for different values of $g$. When $g=0$ as shown in Fig. 6(a), the dynamics represent that of the anharmonically driven plasma oscillation as presented by Enjieu Kadji et al. ${ }^{35}$. With increase in the value of $g$, from $g=0$ to 500 , the system's dynamics becomes largely dominated by the fast oscillation; so that 
oscillations due to the fast input signal now modulate the weak signal. Consequently, a change is induced in the system's response as illustrated Fig. 6 - the consequence being a modification to the system's potential such that its effective potential now determines the resonant state.

Finally, the dependencies of both the response amplitude $Q$ and phase shift $\phi$ on the amplitude of the input signal at fixed dissipation coefficient $\varepsilon$ were also examined. The results are presented in Fig. 7 for different values of low frequencies $\omega(0.05,0.1,0.25$ and 0.5$)$ and forcing amplitudes $f(0.05,0.5,5,15)$ with fixed parameters $\delta=0.3, \kappa=1.0, \omega_{0}=1, \varepsilon=0.02$. Again, we observe the occurrence of both single vibrational resonance (Fig. 7(b)) and bi-resonances (Fig. 7(a)) with respect to $g$. Besides the dependence of the resonance peaks on the value of $g$, there is slight shift in the values of $g$ at which resonance occur for different values of $\omega$.

\section{CONCLUSIONS}

In summary, we have examined and analyzed vibrational resonance in a plasma oscillator under the influence of two periodic driving forces. We analytically derived the equation for the slow motion in terms of the parameters of the fast signal and examined the contributions of the nonlinear dissipation to the system's dynamics. The equation of the slow motion reveals that the contribution from the fast signal to the dissipation of the system is such that the effective nonlinear dissipation of the system play a similar role as the effective potential. We carried out numerical simulations to investigate the effects of nonlinear dissipation on the system's dynamics and verify the existence of VR. Beside the dynamical changes, which includes multiple symmetry-breaking bifurcations, attractor escapes, and reversed period-doubling bifurcations that were not reported previously, we found that high frequency external forcing is indeed capable of inducing both single and double resonances. This carries some direct industrial applications in signal or output filtering control as well as enhancement which include detecting, extracting, or separating signals, reducing noise, or accentuating certain features of the plasma signal. Moreover, Plasma plays major roles in the production of integrated circuits consisting of repeated steps of deposition, masking, etching, and stripping to form and connect circuit elements like transistors and capacitors ${ }^{32}$. In this application, VR can be employed in the production of dense plasma which can increase the etch or deposition rate from different sources. An ideal plasma source possesses straight ion orbits with controllable energy. For profile control, ions are accelerated in a sheath, whose voltage drop is set by the DC potential applied to the etched surface. The DC potential is created by applying an Radio-frequency (RF) voltage to the substrate and using the unidirectional electron flow to charge the substrate negatively. In parallel-plate discharges, this RF bias cannot be controlled independently, since increas- ing the applied RF voltage increases the density and other parameters also, resulting in too large ion energy. In inductive and Electron Cyclotron Resonance (ECR) sources, the plasma is ionized independently of the substrate's RF bias with slow transport process, and ion energies of the desired value can be applied as the fast input signal. In this manner, enhancement, control or filtering of desired responses in plasma production can thus be achieved. Finally, a detailed investigation of the parameter regime for which VR may occur in system (25) would be an interesting direction for future work.

\section{ACKNOWLEDGMENTS}

UEV is supported by the Royal Society of London, through their Newton International Fellowship Alumni scheme. The authors would like to acknowledge the reviewers and editorial office for their constructive criticisms that improved the quality this paper tremendously.

${ }^{1}$ S. Rajasekar and M. A. F. Sanjuan, Nonlinear Resonances, Springer Series in Synergetics (Springer, Switzerland, 2016).

${ }^{2}$ P. S. Landa and P. V. E. McClintock, "Vibrational resonance," Journal of Physics A: Mathematical and General 33, L433 (2000).

${ }^{3} \mathrm{Q}$. Li and R. Zhu, "Stochastic resonance in delayed two-coupled oscillators without common perturbations," Physical Review E 64 (2001).

${ }^{4}$ J. Casado-Pascual, J. Gómez-Ordónez, and M. Morillo, "Stochastic resonance: theory and numerics." Chaos 15, 26115 (2005).

${ }^{5}$ R. Yuan, X. Wang, Y. Ma, B. Yuan, and P. Ao, "Exploring a noisy Van der Pol type oscillator with a stochastic approach." Physical review. E, Statistical, nonlinear, and soft matter physics 87, 062109 (2013).

${ }^{6}$ S. Saikia, A. M. Jayannavar, and M. C. Mahato, "Stochastic resonance in periodic potentials." Physical review. E, Statistical, nonlinear, and soft matter physics 83, 061121 (2011).

${ }^{7} \mathrm{~J}$. Li, "Enhancement and weakening of stochastic resonance for a coupled system," Chaos: An Interdisciplinary Journal of Nonlinear Science 21, 043115 (2011).

${ }^{8}$ L. Chew, C. Ting, and C. Lai, "Chaotic resonance: Two-state model with chaos-induced escape over potential barrier," Physical Review E $\mathbf{7 2}$ (2005).

${ }^{9}$ I. Tokuda, C. Han, K. Aihara, M. Kawato, and N. Schweighofer, "The role of chaotic resonance in cerebellar learning." Neural networks 23, 83642 (2010).

${ }^{10}$ M. Gitterman, "Bistable oscillator driven by two periodic fields," Journal of Physics A: Mathematical and General 34, L355 (2001).

${ }^{11}$ I. Blekhman and P. Landa, "Conjugate resonances and bifurcations in nonlinear systems under biharmonical excitation," International Journal of Non-Linear Mechanics 39, 421 - 426 (2004).

${ }^{12}$ I. Blechman, Vibrational Mechanics (World Scientific,Singapore, 2000).

${ }^{13}$ V. N. Chizhevsky, "Vibrational higher-order resonances in an overdamped bistable system with biharmonic excitation," Phys. Rev. E 90, 042924 (2014).

${ }^{14}$ S. Rajasekar, K. Abirami, and M. A. F. Sanjuan, "Novel vibrational resonance in multistable systems," Chaos 21, 033106 (2011).

${ }^{15}$ J. H. Yang and X. B. Liu, "Controlling vibrational resonance in a multistable system by time delay," Chaos 20, 033124 (2010).

${ }^{16}$ V. N. Chizhevsky, "Experimental evidence of vibrational resonance in a multistable system," Phys. Rev. E 89, 062914 (2014). 
${ }^{17}$ B. Deng, J. Wang, X. Wei, H. Yu, and H. Li, "Theoretical analysis of vibrational resonance in a neuron model near a bifurcation point," Phys. Rev. E 89, 062916 (2014).

${ }^{18} \mathrm{M}$. Borromeo and F. Marchesoni, "Vibrational ratchets," Phys. Rev. E 73, 016142 (2006).

${ }^{19} \mathrm{~S}$. Jeyakumari, V. Chinnathambi, S. Rajasekar, and M. A. F. Sanjuan, "Analysis of vibrational resonance in a quintic oscillator," Chaos 19, 043128 (2009).

${ }^{20} \mathrm{~J}$. H. Yang and X. B. Liu, "Controlling vibrational resonance in a delayed multistable system driven by an amplitude-modulated signal," Physica Scripta 82, 025006 (2010).

${ }^{21}$ C. Jeevarathinam, S. Rajasekar, and M. A. F. Sanjuan, "Theory and numerics of vibrational resonance in Duffing oscillators with time-delayed feedback," Phys. Rev. E 83, 066205 (2011).

${ }^{22}$ C. Jeevarathinam, S. Rajasekar, and M. A. F. Sanjuán, "Vibrational resonance in the Duffing oscillator with distributed timedelayed feedback," arXiv:1504.04163v1 [nlin.CD] (2015).

${ }^{23}$ S. Jeyakumari, V. Chinnathambi, S. Rajasekar, and M. A. F. Sanjuan, "Vibrational resonance in an asymmetric Duffing oscillator," International Journal of Bifurcation and Chaos 21, 275286 (2011).

${ }^{24}$ J. Yang and H. Zhu, "Vibrational resonance in Duffing systems with fractional-order damping," Chaos: An Interdisciplinary Journal of Nonlinear Science 22, 013112 (2012).

${ }^{25}$ Y. Qin, J. Wang, C. Men, B. Deng, and X. Wei, "Vibrational resonance in feedforward network," Chaos 21, 023133 (2011).

${ }^{26}$ B. Deng, J. Wang, X. Wei, K. M. Tsang, and W. L. Chan, "Vibrational resonance in neuron populations," Chaos 20, 013113 (2010).

${ }^{27}$ H. Yu, J. Wang, C. Liu, B. Deng, and X. Wei, "Vibrational resonance in excitable neuronal systems," Chaos 21, 043101 (2011).

${ }^{28} \mathrm{X}$. Wu, C. Yao, and J. Shuai, "Enhanced multiple vibrational resonances by $\mathrm{Na}+$ and $\mathrm{K}+$ dynamics in a neuron model," Scientific reports $\mathbf{5}$ (2015).

${ }^{29}$ J. Shi, C. Huang, T. Dong, and X. Zhang, "High-frequency and low-frequency effects on vibrational resonance in a synthetic gene network." Physical Biology 7, 036006 (2010).

${ }^{30}$ S. Rajasekar, J. Used, A. Wagemakers, and M. Sanjuan, "Vibrational resonance in biological nonlinear maps," Communications in Nonlinear Science and Numerical Simulation 17, $3435-3445$ (2012).

${ }^{31}$ J. P. Baltanás, L. López, I. I. Blechman, P. S. Landa, A. Zaikin, J. Kurths, and M. A. F. Sanjuán, "Experimental evidence, numerics, and theory of vibrational resonance in bistable systems," Phys. Rev. E 67, 066119 (2003).

${ }^{32}$ F. F. Chen, "Industrial applications of low temperature plasma Physics," Physics of Plasmas 2, 2164 (1995).
${ }^{33}$ A. Niknam, D. Komaizi, and M. Hashemzadeh, "Simulation of low frequency buneman instability of a current-driven plasma by particle in cell method," Physics of Plasmas 18, 022301 (2011).

${ }^{34}$ M. Siewe Siewe, F. Moukam Kakmeni, C. Tchawoua, and P. Woafo, "Nonlinear response, and homoclinic chaos of driven charge density in plasma," Report 39090566 (International Atomic Energy Agency (IAEA), Abdus Salam International Centre for Theoretical Physics, Trieste (Italy), 2007).

${ }^{35}$ H. G. Enjieu Kadji, B. R. Nana Nbendjo, J. B. Chabi Orou, and P. K. Talla, "Nonlinear dynamics of plasma oscillations modeled by an anharmonic oscillator," Physics of Plasmas 15, 032308 (2008).

${ }^{36}$ C. N. D. Buckjohn, M. S. Siewe, C. Tchawoua, and T. C. Kofane, "Transition to chaos in plasma density with asymmetry doublewell potential for parametric and external harmonic oscillations," International Journal of Bifurcation and Chaos 21, 1879-1893 (2011).

${ }^{37}$ H. G. Enjieu-Kadji and B. R. Nana-Nbendjo, "Passive aerodynamics control of plasma instabilities," Communications in Nonlinear Science and Numerical Simulations 17, 1779-1794 (2012).

${ }^{38}$ U. E. Vincent, S. O. Kareem, B. R. Nana Nbendjo, and A. N. Njah, "Quasi-synchronization dynamics of coupled and driven plasma oscillators," Chaos, Solitons and Fractals 70, 85 - 94 (2015).

${ }^{39}$ H. G. Enjieu-Kadji, J. B. Chabi-Orou, and P. Woafo, "Regular and chaotic behaviors of plasma oscillations modeled by a modified Duffing equation," Physica Scripta 77, 025503 (2008).

${ }^{40}$ U. Parlitz and W. Lauterborn, "Superstructure in the bifurcation set of the Duffing equation," Phys. Lett. A 107A, 351-355 (1985).

${ }^{41}$ J. Kozłowski, U. Parlitz, and W. Lauterborn, "Bifurcation analysis of two coupled periodically driven Duffing oscillators," Phys. Rev. E 51, 1861-1867 (1995).

${ }^{42}$ U. E. Vincent and A. Kenfack, "Synchronization and bifurcation structures in coupled periodically forced non-identical Duffing oscillators," Physica Scripta 77, 045005 (2008).

${ }^{43}$ D. G. Anishchenko, V. S.and Luchinsky, P. V. E. McClintock, I. A. Khovanov, and N. A. Khovanova, "Fluctuational escape from a quasi-hyperbolic attractor in the Lorenz system," J. Exp. Theor. Phys. 94, 821-833 (2002).

${ }^{44}$ Z. Chen, Y. Li, and X. Liu, "Noise induced escape from a nonhyperbolic chaotic attractor of a periodically driven nonlinear oscillator," Chaos 26, 063112 (2016). 